Criar Educação, Criciúma, v. 10, no1, jan/jul 2021.- PPGE - UNESC - ISSN 2317-2452

\title{
OS IMPACTOS DAS POLÍTICAS PÚBLICAS NO COTIDIANO ESCOLAR
}

\author{
Joselaine Andréia de Godoy Stênico ${ }^{1}$
}

\section{RESUMO}

Da política educacional à prática pedagógica, um elemento está sempre presente, a escola como uma instituição com cultura própria. Assim, o modo como a escola trata a aplicabilidade das políticas públicas de educação acontece sempre de maneira dinâmica e não de forma estanque, interferindo diretamente na cultura escolar, impactando diretamente na organização e desenvolvimentos dos trabalhos e das práticas docentes. Desse modo, este artigo tem como objetivo investigar os reflexos que as políticas públicas produzem no cotidiano escolar, sobretudo, no que se refere aos impactos na organização do trabalho na escola e na configuração da cultura escolar. Trata-se de um estudo empírico com abordagem qualitativa que utilizou a análise bibliográfica como opção metodológica. Entre os resultados obtidos, vale ressaltar que a maioria das políticas públicas educacionais não apenas impactam o cotidiano das escolas no que se refere à formação cognitiva dos sujeitos, mas concentram-se nas mudanças provocadas na cultura escolar, geralmente relacionadas às práticas educativas, à organização e desenvolvimento do trabalho docente, produzindo intensos reflexos no tipo de cidadãos inseridos em um contexto social específico que a escola pretende formar. Desse modo, as políticas públicas voltadas ao campo educacional deveriam adequar suas proposições em função das reais demandas da escola.

Palavras-Chave: Política Pública. Cultura Escolar. Trabalho Docente.

\section{THE IMPACTS OF PUBLIC POLICIES IN THE SCHOOL DAILY}

\section{ABSTRACT}

From educational policy to pedagogical practice, an element is always present, the school as an institution with its own culture. Thus, the way in which the school treats the applicability of public policies of education always happens in a dynamic way and not in a sealed way, directly interfering in the school culture, directly impacting the organization and development of the works and the teaching practices. In this way, this article aims to investigate the reflexes that the public policies produce in the school routine, above all, in what concerns the impacts on the organization of the work in the school and the configuration of the school culture. This is an empirical study with a qualitative approach that used bibliographic analysis as a methodological option. Among the results obtained, it is worth mentioning that most public educational policies not only impact the daily life of schools in relation to the cognitive training of the subjects, but are focused on the changes provoked in the school culture,

1 Doutora em Educação pela Universidade Estadual Paulista "Júlio de Mesquita Filho" UNESP Campus de Rio Claro/SP (2019), Mestre em Educação pela UNESP (2014). 
Criar Educação, Criciúma, v. 10, no1, jan/jul 2021.- PPGE - UNESC - ISSN 2317-2452

generally related to educational practices, organization and development of the teaching work, producing intense reflexes in the type of citizens inserted in a specific social context that the school intends to form. In this way, the public policies directed to the educational field should adapt their propositions in function of the real demands of the school.

Keywords: Public Policy. School Culture. Teaching Work.

\section{INTRODUÇÃO}

É papel da instituição escolar e dos educadores preocupar-se, igualmente, com a aquisição de conhecimentos científicos e com uma formação cidadã, na qual o sujeito fosse preparado para se tornar um agente social que, munido de informações relevantes, fosse capaz de refletir e de emitir juízo a respeito da situação social, política e econômica em que está inserido, reconhecendo o seu papel na construção histórica da democracia e se tornando apto para idealizar sua vida pessoal, bem como sua vida em sociedade.

A instituição educacional se encontra inserida nas prerrogativas legais e, portanto, os preceitos legais subsidiam o processo educativo. As políticas educacionais adequam as atividades escolares e a sua forma de aprendizado. Essas políticas ao serem aplicadas no ambiente escolar, por vezes, impactam na construção do cotidiano e dos atores educacionais.

A Constituição Brasileira de 1988 garante a educação como um dos direitos constituídos legalmente em seu Art. 205 ressalta que "a educação, direito de todos e dever do Estado e da família". No Art. 206 afirma que o ensino será ministrado com base em diversos princípios, entre eles, cabe destaque para a gratuidade do ensino público nos estabelecimentos oficiais e também garantia de padrão de qualidade.

O Art. 227 especifica que é dever da família, da sociedade e do Estado assegurar à criança e ao adolescente, com absoluta prioridade, o direito à vida, à saúde, à alimentação, à educação, ao lazer, à profissionalização, à cultura, à dignidade, ao respeito, à liberdade e à convivência familiar e comunitária, além de colocá-los a salvo de toda forma de negligência, discriminação, exploração, violência, crueldade e opressão.

O Art. 208 explicita o dever do Estado com a educação: $\S 2^{\circ}$ O não 
Criar Educação, Criciúma, v. 10, no1, jan/jul 2021.- PPGE - UNESC - ISSN 2317-2452

oferecimento do ensino obrigatório pelo Poder Público, ou sua oferta irregular, importa responsabilidade da autoridade competente.

$\mathrm{Na}$ Lei de Diretrizes e Bases da Educação Nacional, doravante LDB, Lei no 9.394, de 20 de dezembro de 1996, afirma no título III, Art. 4º que é dever do Estado com a educação escolar pública a garantia do ensino fundamental, obrigatório e gratuito, inclusive, para os que a ele não tiveram acesso na idade própria; progressiva extensão da obrigatoriedade e gratuidade ao ensino médio; atendimento gratuito em creches e pré-escolas.

Diante dessas evidências legais, nota-se, portanto, que a educação é um direito estendido a todos os cidadãos brasileiros, sendo dever do Estado a sua garantia, daí a necessidade de elaboração e implantação de políticas públicas no campo da educação.

Cabe ao Governo assegurar determinados direitos aos cidadãos, a exemplo de direitos fundamentais sociais, como saúde, educação, segurança pública. O Executivo não apenas executam as leis, ele cria suas próprias políticas e programas necessários à realização dos ordenamentos legais. Esses direitos são viabilizados aos cidadãos através de políticas públicas (SILVA, 2008, p. 03).

Saut (2007) explica que as políticas públicas representam um ponto fundamental e necessário para a garantia dos direitos, porque integram o conceito e a função social do Estado, assim como constituem a primeira instância de soluções, possibilidades e oportunidades de transformação da realidade.

No cerne dessa proposição, este artigo investiga os reflexos que as políticas públicas produzem no cotidiano escolar, sobretudo, no que se refere aos impactos na organização do trabalho na escola e na configuração da cultura escolar.

\section{PROCEDIMENTOS METODOLÓGICOS}

Para atingir os objetivos a que nos propomos, realizou-se um estudo que abrange uma investigação empírica com abordagem de uma pesquisa qualitativa, que tem como principal finalidade: 
Criar Educação, Criciúma, v. 10, no1, jan/jul 2021.- PPGE - UNESC - ISSN 2317-2452

[...] o propósito de explicar alguns aspectos da realidade, para assim, ser possível agir, intervir sobre ela, identificando os problemas, formulando, experimentando, avaliando e aperfeiçoando alternativas de solução, em situação real, com a intenção de contribuir para o aperfeiçoamento contínuo dessa realidade (LIMA, 2004, p. 33).

Rampazzo (2002) ressalta que a pesquisa qualitativa busca estudar um fenômeno em particular com o objetivo de compreendê-lo e não explicá-lo, desse modo, é possível conseguir um significado mais profundo, concebido como um empreendimento mais abrangente e multidimensional.

O autor afirma ainda que o rigor da pesquisa não está na precisão numérica, mas sim em fenômenos que não podem ser estudados quantitativamente, por se tratarem de dimensões pessoais. Não se privilegia o estudo apenas do objeto ou o estudo do sujeito, mas sim a relação entre ambos, desse modo, a pesquisa qualitativa valoriza o sujeito humano que não se pode ser reduzido a um esquema generalizado, quantidades e números.

No que tange à utilização da "Pesquisa Bibliográfica", Tozoni-Reis (2009, p. 42 e 43) afirma que a principal característica desse tipo de metodologia é a busca de conhecimentos sobre os fenômenos investigados por meio de bibliografia especializada, visando “(...) conversar e debater com os autores através de seus escritos (...) concordar, discordar, discutir, problematizar os temas à luz das ideias dos autores".

A autora assinala ainda que as técnicas utilizadas na pesquisa bibliográfica são a leitura sistematizada e o fichamento bibliográfico. Esses processos são considerados importantes, pois permite catalogar e organizar todas as leituras com a finalidade de coletar dados. O resultado desses processos deverá ser empreendido nas análises dos temas em estudo.

Recorreu-se a essa metodologia durante todo o processo de elaboração de pesquisa, pois todas as descrições, análises, debates e interpretações passaram pelo esforço eminente de explorar a literatura na busca de identificar o que foi produzido de conhecimento pela comunidade científica sobre o tema.

Além disso, utilizou-se como opção metodológica a pesquisa bibliográfica, pois buscou-se aprofundamento na temática em questão, por meio de leituras, 
Criar Educação, Criciúma, v. 10, no1, jan/jul 2021.- PPGE - UNESC - ISSN 2317-2452

análises e reflexões da produção de autores diversos que discutem o tema.

Os procedimentos de busca e seleção das bibliografias utilizados na revisão foram pesquisas que usaram a abordagem qualitativa e apontamentos sobre a relação das políticas públicas educacionais e os respectivos impactos no cotidiano da escola.

\section{OS IMPACTOS QUE AS POLÍTICAS PÚBLICAS PROMOVEM NO COTIDIANO ESCOLAR}

É por meio da instituição escolar que o ensino e a aprendizagem de fato tomam corpo, promovendo a educação do sujeito em relação ao conhecimento, tarefas e valores. É ainda através da escola onde efetivamente ocorrem os processos importantes que favorecem o cumprimento das atividades pedagógicas, curriculares e docentes que darão fundamentos para continuidade nos estudos e até mesmo prosseguimento para a entrada no mundo do trabalho.

[...] a escola, semelhante a outras organizações, é lócus de bases conflitivas que, mediante processos históricos, conformam o terreno político e organizativo no qual se move cada comunidade escolar concreta. Este contexto, situado e nutrido em bases conflitivas (MOLINA, LOPES e ACHILLES, 2001, p. 923).

Além dos desafios a ela dirigidos, constantemente as escolas precisam adequar-se a implementação de políticas educacionais. Toda unidade escolar sofre, direta ou indiretamente, os impactos advindos das políticas públicas educacionais, por conseguinte, a apropriação de novos contextos direcionados pelas políticas altera a cultura escolar.

Molina, Lopes e Achilles, (2001, p. 923) explicam que a legislação frequentemente constitui-se em fontes de esperança, pois as práticas sociais, políticas, éticas e culturais fundam-se em valores construídos nos processos sociais e na cultura dessas comunidades, entretanto, embora se apresente como algo inovador, frequentemente, há "(...) manutenção de narrativas antigas e particulares da escola e pouco inovadoras em relação ao modelo tradicional". 
Criar Educação, Criciúma, v. 10, no1, jan/jul 2021.- PPGE - UNESC - ISSN 2317-2452

O protagonismo dos docentes é fundamental para o movimento da dinâmica da organização escolar, pois "(...) interagem e produzem efeitos na organização escolar conformando-a e ao mesmo tempo são conformados por ela" (MOLINA, LOPES e ACHILLES, 2001, p. 936), favorecendo, assim, novas significações no processo identitário dos docentes.

Ferreira e Nogueira (2015), por sua vez, explicam que as políticas públicas provocam alterações no interior da escola, sobretudo, no que se refere à forma de organização e desenvolvimentos dos trabalhos, o que afeta diretamente as práticas docentes.

Os autores ressaltam ainda que a legislação por si só, não altera a realidade escolar, ela só repercutirá no cotidiano das escolas desde que se tenha um ambiente propício a alterações, com interferência na cultura escolar.

Souza (2003) ao falar dos impactos das políticas de avaliação no desenvolvimento do currículo escolar afirma que não se pode abordar de modo abstrato, antes é necessário situar o procedimento ou processo que é tomado como referência e quais a finalidades e contornos de que ela se reveste.

No caso das políticas de avaliação, a autora explica que os impactos nos sistemas escolares estão voltados mais especificamente na difusão de uma concepção de avaliação que estimula a competição entre as escolas, responsabilizando-as pelo sucesso ou fracasso escolar do que a busca de subsídios para intervenções mais precisas e consistentes do poder público na educação básica.

A autora explica que a aceitação das avaliações não tem potencial para produzir alterações nas práticas escolares, de ensino e de aprendizagem apenas fortalece o papel regulador do Estado, por meio da responsabilização das unidades federadas pelos resultados escolares.

Delgado (2012) ao citar a implantação da política de promoção automática, sobretudo, no Estado de São Paulo que se deu por meio da Deliberação do Conselho Estadual de Educação - CEE - n 09/97, afirma que termos como, ciclos, progressão continuada, promoção automática, aulas de reforço, recuperação 
Criar Educação, Criciúma, v. 10, no1, jan/jul 2021.- PPGE - UNESC - ISSN 2317-2452

paralela, programas de aceleração, avaliação contínua, passaram a fazer parte do cotidiano das escolas.

A implantação dessa política pública na época, segundo a autora, teve grande impacto no sistema de ensino, sobretudo, no ensino fundamental e gerou ansiedades e controvérsias no interior das escolas.

[...] quando há uma proposta advinda de uma política pública que incide diretamente nas práticas escolares, não se pode analisá- la e buscar compreendê- la isoladamente e sim a partir de um olhar mais amplo que considere todos os fatores que intervêm e possibilitam, ou não, a consecução dessa proposta. Ou seja, é preciso considerar como os espaços e os tempos se organizam, o currículo escolar e as condições de oferta do ensino nas escolas, como por exemplo, o número de alunos por sala. E também, a formação do professor, suas práticas e a postura dos seus dirigentes e demais agentes educacionais que interferem nas mudanças pretendidas pelas políticas públicas (DELGADO, 2012, p. 164).

A autora explica que talvez pelo fato das políticas públicas desconhecerem o cotidiano escolar, há um descompasso enorme entre o dito (proclames legais) e o feito (o que de fato acontece no chão da escola), nesse sentido, Delgado (2012) advoga que as políticas públicas deveriam adequar suas proposições em função das reais demandas da escola, e não o contrário.

Nesse contexto, de acordo com Oliveira (2003, p. 33) "(...) o movimento de reformas (...) traz consequências significativas para a organização e a gestão escolar, resultando uma reestruturação do trabalho docente, podendo alterar até sua natureza".

Complementarmente, Alvarenga, Vieira e Lima (2006) explicam que as políticas públicas implementadas pelo governo na educação evidenciam a necessidade de adequação do trabalho docente às novas exigências. O Estado intervém no trabalho docente de modo a instituir mecanismos que induzam os docentes a adequarem as atividades inerentes à profissão de acordo com os resultados estabelecidos pelos interesses mercantilistas.

Assim, por meio das políticas educacionais, o trabalho docente tende a sofrer consequências importantes em função de sua natureza, dos objetivos e finalidades. 
Observa-se também um acúmulo de responsabilidades quanto aos procedimentos do/a professor/a, tornando sua rotina absorvida por tarefas que impedem uma prática refletida, profundamente articulada com a prática social, transformando tais profissionais em práticos que repetem os procedimentos aprendidos na formação inicial, muitas vezes insuficientes para responder às questões colocadas pelo contexto em que atuam, quanto à sua atuação política e pedagógica. Tudo isso agravado pelo fato de que são os/as professores/as responsabilizados individualmente por seus sucessos e fracassos profissionais, pelas dificuldades e pela qualidade do ensino, bem ao gosto da ideologia liberal vigente. Ausentes do trabalho de planejamento e de elaboração de projetos, currículos e avaliação, há uma simplificação/empobrecimento da função docente, tornando seu trabalho cada vez mais a mercê da interferência do Estado, que amplia seu uso dos mecanismos persuasivos da sociedade civil, ampliando a hegemonia da classe que representa (ALVARENGA, VIEIRA e LIMA, 2006, p. 04).

As autoras afirmam que as políticas públicas implantadas no campo educacional tem se ajustado à globalização da economia. Esse processo trouxe impacto direto no campo educacional, sobretudo, no que se refere às políticas de formação docentes.

As políticas sob a bandeira de modernização dos processos educativos e democratização do acesso à formação dos milhares de professores/as da educação básica no país, tem resultado em um saldo negativo na qualidade da formação do magistério.

As autoras explicam ainda, que essas propostas colocam o professor como um elemento facilitador das reformas e que por si só, conseguem transformar o cotidiano escolar, responsabilizando-os, assim, pelos insucessos da escola.

Sobre isso, Novaes (2009, p. 19) explica que esse discurso valoriza "(...) a qualidade como sinônimo de produtividade, que subordina autonomia aos conceitos de eficiência e eficácia, que utiliza os conceitos de descentralização e desconcentração como pretextos para a responsabilização individual dos envolvidos no trabalho escolar".

\section{A CULTURA ESCOLAR E AS POLÍTICAS PÚBLICAS}

$\mathrm{Na}$ seção anterior foi possível observar alguns impactos que as políticas públicas promovem no cotidiano escolar. A maioria delas se concentra nas mudanças na cultura escolar, geralmente relacionadas às práticas educativas, à 
Criar Educação, Criciúma, v. 10, no1, jan/jul 2021.- PPGE - UNESC - ISSN 2317-2452

organização e desenvolvimentos dos trabalhos docentes.

De acordo com Silva (2006), a cultura escolar é a vida interna da escola, constituída pelos valores, práticas e normas, compreendendo o conjunto de saberes que, uma vez organizado, didatizado, compõe a base de conhecimentos sobre a qual trabalham professores e alunos. Julia (2001), por sua vez, define a cultura escolar como:

Um conjunto de normas que definem conhecimentos a ensinar e condutas a inculcar, e um conjunto de práticas que permitem a transmissão desses conhecimentos e a incorporação desses comportamentos; normas e práticas coordenadas as finalidades que podem variar segundo as épocas (finalidades religiosas, sociopolíticas ou simplesmente de socialização) (JULIA, 2001, p. 10).

A cultura escolar perpassa as ações do cotidiano escolar, "(...) seja na influência sobre os seus ritos ou sobre a sua linguagem, seja na determinação das suas formas de organização e de gestão, seja na constituição dos sistemas curriculares" (SILVA, 2006, p. 204).

Atualmente, em curso, estão algumas políticas educativas que certamente afetará as escolas brasileiras, especialmente cabe citar, a Base Nacional Comum Curricular (BNCC). Trata-se de um conjunto de orientações que deverá nortear os currículos das escolas, redes públicas e privadas de ensino de todo o Brasil. A Base trará os conhecimentos essenciais, as competências e as aprendizagens pretendidas para as crianças e jovens em cada etapa da Educação Básica em todo país.

Como se trata de uma política pública ainda em curso, a BNCC foi encaminhada ao Conselho Nacional de Educação (CNE) que elaborou um parecer sobre o tema, em seguida, submeteu ao Ministro da Educação, a quem competiu homologar as deliberações do Conselho para que entrem em vigor, tal fato ocorreu em 14 de dezembro de 2018, quando, então, o ministro da Educação, Rossieli Soares, homologou o documento da BNCC e, sendo assim, o Brasil passou a ter uma Base com as aprendizagens previstas para toda a Educação Básica.

Posteriormente essas etapas, começou-se desde 2018 a implementação da Base, em conjunto com as redes de ensino, que tem e, ainda terão, a tarefa de 
Criar Educação, Criciúma, v. 10, no1, jan/jul 2021.- PPGE - UNESC - ISSN 2317-2452

adequar seus currículos, ou seja, nota-se que essa política afetará diretamente as escolas que deverão desenvolver seus currículos baseados na BNCC. Provavelmente houve e ainda haverá uma mobilização dos gestores, dos professores em torno do novo currículo que certamente implicará em impactos na constituição dos atores da educação que determinará o tipo de sujeito que será formado e que certamente impactará na cultura escolar.

Desse modo, o que se observa é que as políticas públicas voltadas à educação não apenas impactam o cotidiano das escolas no que se refere à formação cognitiva dos sujeitos provocando mudanças na cultura escolar, mas também produz intensos reflexos no tipo de cidadãos inseridos em um contexto social específico que a escola pretende formar.

Complementarmente a isso, assistimos também a transferência de obrigações do Estado para o "terceiro setor", este por último, vale ressaltar que surgiu na segunda metade do século XX, vislumbrando como um espaço social em que instituições privadas cumpririam o papel, de garantir direitos básicos, na forma de serviços de mercado, um papel antes de responsabilidade do Estado.

O que se observou foi uma demanda crescente da utilização de parcerias público-privadas para ampliar a oferta de serviços de educação e cultura à população e, com isso, identificou-se a formulação e implementação das políticas públicas sociais baseada nessas parcerias efetuadas entre o Estado e o Terceiro Setor.

Frente às parcerias efetuadas entre o Estado e o Terceiro Setor, especialmente na área social, com a compra, pelo Estado de pacotes prontos de projetos educacionais provenientes do "Terceiro Setor", cuja forma de implementação local já é pré-determinada em convênios e parcerias, sobra pouco espaço para a participação da sociedade civil na definição, formulação e implementação das políticas públicas sociais hoje, no Brasil (PERONI et al, 2009).

Desse modo, o que se nota é que as escolas e seus atores precisam se adequar as demandas políticas tal como o BNCC e as parcerias públicos-privados, como aqui apresentamos, entretanto, conforme explicita Silva (2006), as escolas 
Criar Educação, Criciúma, v. 10, no1, jan/jul 2021.- PPGE - UNESC - ISSN 2317-2452

podem apresentar diferentes realidades, pois embora tenham características e comportamentos que as aproximam, há outras que as diferenciam. E, com isso, por vezes, nota-se que as mesmas escolas não conseguem atingir as metas esperadas ou não conseguem colocar em prática os discursos oficiais para a materialização no tempo-espaço do cotidiano da escola.

Sobre isso, Julia (2001) explica que a cultura escolar não pode ser estudada sem a análise das relações de conflito em cada período histórico com o conjunto de culturas contemporâneas. Além disso, deve-se considerar ainda que para uma análise efetiva das normas e práticas propostas e realizadas para determinados fins, os professores são elementos fundamentais nessa relação, visto que são os sujeitos encarregados da aplicação das referidas normas e, sendo assim, os modos de pensar e de agir difundidos na sociedade que concebem a aquisição de competências e habilidades pelos processos formais de escolarização são perspectivas que devem ser consideradas.

Isso implica que saberes e habitus requeridos de um futuro professor, tornase objeto de análise da cultura escolar, junto a isso, os conteúdos ensinados e as práticas escolares precisam ser entendidas como um produto específico da escola, evidenciando o caráter criativo do sistema escolar e não apenas como um modo de vulgarizar ou adaptar a ciência.

Contrariamente as ideias recebidas, o estudo histórico das disciplinas escolares mostra que, diante das disposições gerais atribuídas à escola, os professores dispõem de ampla liberdade de manobra: a escola não é o lugar da rotina e da coação e o professor não é o agente de uma didática que Ihe seria imposta de forma [...] ele sempre tem a possibilidade de questionar a natureza de seu ensino (JULIA, 2001, p. 33).

Portanto, impor que uma política seja efetivada de modo semelhante entre as escolas é utópico, pois sendo assim, desconsidera a cultura escolar e os atores das escolas, inclusive, Julia (2001) nos ensina que somente o grupo de alunos que o professor tem diante de si pode exercer algum tipo de restrição, pois é diante desse público que o professor identifica os saberes que funcionam e os que não funcionam e, portanto, "(...) é a mudança de público que impõe frequentemente a mudança dos conteúdos ensinados" (JULIA, 2001, p. 34). 
Criar Educação, Criciúma, v. 10, no1, jan/jul 2021.- PPGE - UNESC - ISSN 2317-2452

O que se nota, portanto, é certo distanciamento da norma prescrita com a cultura escolar. A escola possui suas particularidades e especificidades que nem sempre são consideradas. Forquin (1993) faz uma análise interessante a esse respeito:

A educação escolar não se limita a fazer uma seleção entre os saberes e os materiais culturais disponíveis num dado momento histórico, ela deve também, para torná-los efetivamente transmissíveis, efetivamente assimiláveis às novas gerações, entregar-se a um imenso trabalho de reorganização, de reconstrução, ou de "transposição didática" (FORQUIN, 1993, p. 16).

De acordo com Perrenoud (1993), a escola submete os saberes, as práticas e as culturas a um conjunto de transformações para que se torne possível ensiná-los, nesse processo, cada professor organiza conhecimentos diversos, seguindo o movimento de sua própria formação.

O autor afirma que a cultura escolar está relacionada a atuação do professor, considerando que "(...) ensinar é, antes de mais nada, fabricar artesanalmente os saberes, tornando-os ensináveis, exercitáveis e passíveis de avaliação no quadro de uma turma, de um ano, de um horário, de um sistema de comunicação e trabalho" (PERRENOUD, 1993, p. 25).

A partir dessas afirmações, uma possível análise que se pode realizar é que as escolas, que detém uma cultura própria e compreendendo as interfaces da cultura e do currículo, pode-se construir e implantar uma política educacional condizente com a realidade da unidade escolar, entretanto, a escola, ao mesmo tempo em que, deve responder aos desafios das políticas públicas, deve também atender a realidade que a circunda, considerando o pleno desenvolvimento da pessoa, seu preparo para o exercício da cidadania, incluindo a formação ética e o desenvolvimento da autonomia intelectual e do pensamento crítico.

Assim, é um desafio enorme para as escolas atender às demandas políticas, sociais e intelectuais sem dissociar a formação geral. Trata-se, portanto, de um processo de contínuo de aprendizado, de ajuda, de desenvolvimento e de reflexões para melhor compreender a si e ao mundo e, nessa trama, os atores educacionais têm papel fundamental para fomentar práticas inovadoras através de um currículo 
Criar Educação, Criciúma, v. 10, no1, jan/jul 2021.- PPGE - UNESC - ISSN 2317-2452

dinâmico e flexível.

\section{Considerações Finais}

Este artigo apresentou os meandros da relação cultura escolar e as políticas públicas, especificamente, tratou de analisar os impactos das políticas públicas sobre o cotidiano da escola.

De modo geral, evidenciou-se que todos os esforços pedagógicos têm, em última análise, a necessidade de atender as demandas das políticas públicas, entretanto, a escola possui suas especificidades e não se trata apenas de caracterizar os protagonistas que atuam no espaço escolar e relacioná-los às necessidades políticas, por conta disso, observa-se certo distanciamento da norma prescrita com a cultura escolar.

Desse modo, notou-se que os conceitos dispostos na relação políticas públicas e cultura escolar demonstram um contexto complexo, no qual a escola está em constante movimento e transformação e, portanto, as políticas públicas não impactam apenas o cotidiano escolar, mas também provocam mudanças na cultura e, por sua vez, no tipo de cidadão que será formado.

O que se analisa, portanto, é que o desafio está em realizar as ações na prática, pois as políticas públicas, mesmo obrigatórias por lei, é preciso que o professor se aproprie da proposta e se coloque como protagonista, tratando-se de uma proposta que será desenvolvida na escola e pela escola, considerando a cultura daquela escola, planejado para atender às reais necessidades da unidade escolar. $A$ partir das análises realizadas, remanesce a seguinte reflexão: a cultura escolar consegue responder a todos os apelos das políticas públicas?

\section{REFERÊNCIAS}

ALVARENGA, Elda; VIEIRA, Emília Peixoto; LIMA, Miriam Morelli. Os impactos das políticas educacionais brasileira no trabalho docente. VI Seminário da

REDESTRADO - Regulação Educacional e Trabalho Docente. 06 e 07 de novembro de 2006 - UERJ, Rio de Janeiro/RJ. 2006.

DELGADO, Adriana Patrício. O impacto das políticas públicas nas práticas escolares 
Criar Educação, Criciúma, v. 10, no1, jan/jul 2021.- PPGE - UNESC - ISSN 2317-2452

sob a ótica da avaliação de aprendizagem. Espaço do currículo, v.4, n.2, p.162171, Setembro de 2011 a Março de 2012.

FERREIRA, Luiz Antonio Miguel; NOGUEIRA, Flávia Maria de Barros. Impactos das políticas educacionais no cotidiano das escolas públicas plano nacional de

educação. Revista @rquivo Brasileiro de Educação, Belo Horizonte, vol.3, num.5, jan-jul, 2015.

JULIA, Dominique. A cultura escolar como objeto histórico. Rev. Bras. Hist. Educ, Rio de Janeiro, n. 1, 2001, p. 9-44.

LIMA, Manolita Correia. Monografia: Engenharia da Produção acadêmica. São Paulo: Saraiva, 2004.

OLINA, Rosane Kreusburg; LOPES, Rodrigo Alberto Lopes, ACHILLE, Helena Soares. Impactos das políticas educacionais nas dinâmicas da organização escolar: reflexões na perspectiva do professorado de uma rede municipal. Ensaio: aval. pol. públ. Educ., Rio de Janeiro, v. 19, n. 73, p. 921-940, out./dez. 2011.

NOVAES, Luiz Carlos. Os impactos da política educacional paulista na prática docente e na organização do trabalho pedagógico nas escolas estaduais paulistas na perspectiva dos professores. Jornal de políticas educacionais. $N^{\circ} 5$, Janeirojunho, p. 13-26, 2009.

OLIVEIRA, Dalila Andrade (org.). Reformas Educacionais na América Latina e os trabalhadores docentes. Belo Horizonte, Autêntica, 2003.

PERRENOUD, Phillippe. Práticas pedagógicas e profissão docente: três facetas. In: Práticas pedagógicas, profissão docente e formação: perspectivas sociológicas. Lisboa: Dom Quixote, 1993.

PERONI, Vera et al. Terceira Via, Terceiro Setor e a parceria IAS/sistemas de ensino público no Brasil. In: Educação: Teoria e Prática - v. 19, n.32, jan.-jun.-2009, p.1735. Rio Claro, SP, Brasil.

RAMPAZZO, Lino. Metodologia científica: para alunos dos cursos de graduação e pós-graduação. São Paulo: Edições Loyola, 2005.

SAUT, Roberto Diniz. Direito da criança e do adolescente e sua proteção pela rede de garantias. Revista Jurídica - CCJ/FURB, v. 11, n. 21, p. 45-73, jan./jun. 2007.

SILVA, Fabiany de Cássia Tavares. Cultura escolar: quadro conceitual e possibilidades de pesquisa. Educar, Curitiba, n. 28, p. 201-216, 2006.

SILVA, Alessandra Obara Soares. Inexistência ou ineficiência das políticas públicas e 

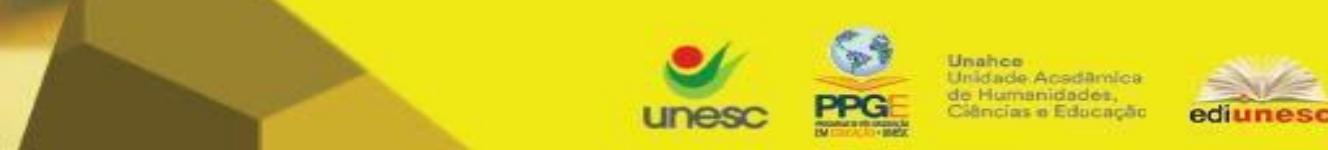

Criar Educação, Criciúma, v. 10, nำ1, jan/jul 2021.- PPGE - UNESC - ISSN 2317-2452

controle judicial. Revista Eletrônica da Faculdade de direito da PUC, São Paulo, v. 1, p.1-22, 2008.

SOUSA, Sandra M. Zákia L. Possíveis impactos das políticas de avaliação no currículo escolar. Cadernos de Pesquisa, n. 119, julho/ 2003.

Recebido setembro de 2020

Aprovado fevereiro de 2021 